\title{
Penetrating the Barriers to Successful $\alpha$-Radioimmunotherapy
}

Sean Carlin, $\mathrm{PhD}$

Department of Radiology, University of Pennsylvania, Philadelphia, Pennsylvania

For "up an' down an' round," said 'e, "goes all appointed things, An' losses on the roundabouts means profits on the swings!"

—Patrick R. Chalmers, "Roundabouts and Swings," 1914

$\mathbf{T}$ here have been many significant advances in the field of radioimmunotherapy since the initial clinical studies were conducted in the 1980s. The emergence of monoclonal antibody technology, high-specific-activity labeling chemistries, and molecular engineering techniques have addressed several of the initial limitations encountered (1), resulting in the first Food and Drug Administration approvals of radioimmunotherapy with $\beta$-particleemitting isotopes ( $\beta$-radioimmunotherapy) for lymphoma.

The utility of $\beta$-radioimmunotherapy, however, is restricted largely to hematologic malignancy, with limited success reported in solid tumors. A central issue for conventional systemic $\beta$-radioimmunotherapy of bulky solid tumors is the therapeutic index, in that there is now a sufficient body of evidence to conclude that, irrespective of radionuclide used or antigen targeted, antitumor efficacy is observed only at doses that result in significant toxicity, principally to bone marrow and liver. This inadequacy results from some inherent features of $\beta$-radioimmunotherapy: in addition to poor antibody penetration, the slow blood clearance of antibody, combined with the millimeterto-centimeter range of the energetic emission from radioactive decay, culminates in increased background absorbed doses and consequently reduced tumor-to-background ratio. A second limiting factor in this setting is that the antitumor efficacy of a given $\beta$-radioimmunotherapy dose decreases with tumor size, because of the proportional increase in the correctly targeted radiation dose that is deposited outside the tumor volume and therefore wasted (2). Essentially, long-range $\beta$-radioimmunotherapy becomes less effective as solid tumors get smaller. Despite these shortcomings, the potential to specifically target occult metastatic lesions on the basis of tumor antigen expression makes alternative radioimmunotherapy strategies worthy of exploration. The use of pretargeting strategies designed primarily to reduce normal-tissue dose are seeing renewed interest because of the emergence of a new generation of chemical components $(3,4)$.

Received Feb. 14, 2018; revision accepted Feb. 21, 2018.

For correspondence or reprints contact: Sean Carlin, Department of Radiology, University of Pennsylvania, 3620 Hamilton Walk, Philadelphia, PA 19129.

E-mail: carlins@mail.med.upenn.edu

Published online Mar. 15, 2018.

COPYRIGHT (c) 2018 by the Society of Nuclear Medicine and Molecular Imaging. DOI: 10.2967/jnumed.117.205146
An article by Palm et al. (5) in the April issue of The Journal of Nuclear Medicine focused on the implementation of radioimmunotherapy with $\alpha$-particle-emitting radionuclides ( $\alpha$-radioimmunotherapy). The rationale for the use of $\alpha$-radioimmunotherapy is based principally on the greater cytotoxicity and shorter range of the $\alpha$-particle than of the $\beta$-particle, resulting in increased antitumor efficacy combined with decreased normal-tissue toxicity. This rationale was recently demonstrated with the success of ${ }^{223} \mathrm{Ra}$ dichloride (Xofigo; Bayer) for prostate cancer metastatic to bone (6), versus the minimal survival benefit reported previously from a range of similarly targeted $\beta$-isotopes $\left({ }^{32} \mathrm{P},{ }^{89} \mathrm{Sr},{ }^{153} \mathrm{Sm}\right)(7)$. Recent reports of dramatic prostate cancer responses to ${ }^{213} \mathrm{Bi}$ - and ${ }^{225} \mathrm{Ac}$-prostate-specific-membrane antigen small-molecule compounds $(8,9)$ also lend support to the case for targeted $\alpha$-particle therapy, but as yet no information on renal toxicity (likely to be dose-limiting) in these cases is available.

$\alpha$-radioimmunotherapy is optimally suited to single-cell, micrometastatic, and minimal residual disease because of 2 governing factors: the poor tumor penetration of intact $\mathrm{IgG}$ and the short $\alpha$-particle range $(\sim 80-100 \mu \mathrm{m})$. For lesions of these dimensions $(<100 \mu \mathrm{m}$ in diameter), antibody binding to only the outer cell layers still places all malignant cells within the $\alpha$-particle range. However, in bulkier disease conformations, the poor penetration of IgG into the tumor mass results in heterogeneous antibody (and radiation dose) distribution and consequent underdosing of nontargeted tumor cells. Tumor penetration of IgG is influenced by a combination of factors, including tight gap junctions (elegantly demonstrated by Sutherland et al. using autoradiography of avascular multicellular spheroids (10)), the physical barrier presented by tumor desmoplastic stroma, and the "binding-site barrier" first proposed by Fujimori et al. (11), a term that describes the phenomenon of reduced tumor diffusion of high-affinity antibodies resulting from the low antibody-antigen dissociation rate. This low rate, combined with abundant local target antigen, results in persistent antibody localization in the region of antibody delivery (usually perivascular) and inhibition of antibody dissemination throughout the tumor. The consequences of nonuniform antibody (and thus radiation dose) distribution, thoroughly examined by O'Donoghue (12), are common for all targeted radionuclides and have increasingly important ramifications as the tumor volume increases relative to the particle range. Put simply, short-range $\alpha$-radioimmunotherapy becomes less effective as solid tumors get larger.

From this fact, it is easy to conclude that there can be no best single antibody-radioisotope pairing for the treatment of both large ( $>\sim 1 \mathrm{~mm}$ in radius) and small $(<50 \mu \mathrm{m}$ in radius) tumor deposits and that the selection of either $\alpha$ - or $\beta$-emitting isotopes will result in increased efficacy in one disease conformation at the 
expense of the other. Gains on the swings mean losses on the roundabouts.

In the study by Palm et al. (5), a combined kinetic and dosimetric model is used to identify methods that maximize $\alpha$-particle therapy for a larger tumor size range, based on short-range $\alpha$-radioimmunotherapy with ${ }^{211}$ At-MX35 (5). The model itself contains many parameters derived from the previous extensive clinical study of the intraperitoneal administration of ${ }^{211}$ Atlabeled MX35 and fragments thereof, recognizing a cell-surface antigen expressed on $90 \%$ of human ovarian epithelial cancers $(13,14) .{ }^{211} \mathrm{At}$ is an $\alpha$-emitting radiohalide with a half-life of $7.2 \mathrm{~h}$, $100 \% \alpha$-particle yield per decay, and no significantly problematic daughter isotopes.

The model presented recapitulates in detail many of the issues outlined above $-\alpha$-particle sterilization of tumors with radii larger than about $50 \mu \mathrm{m}$ relies on antibody diffusion to the subsurface layers, and the inherent poor penetration of IgG over the short half-life of ${ }^{211}$ At results in heterogeneous distribution and subsequent underdosing to central subregions of these larger deposits.

One seemingly straightforward means to overcome the bindingsite-barrier issue for $\alpha$-radioimmunotherapy in larger tumors is via the mass effect. This involves preloading with a large dose of unlabeled antibody to saturate the easily available tumor binding sites and thus facilitate improved tumor penetration of the $\alpha$-radioimmunotherapy. This approach has proven effective, and is routinely used, for some $\beta$-radioimmunotherapy strategies (15-17). However, the study by Palm et al. (5) revealed that overcoming the tumor penetration issue in this manner also reduces absorbed doses in correctly targeted microtumors $(<50 \mu \mathrm{m}$ in radius) to subtherapeutic levels, because of lowered absolute $\alpha$-radioimmunotherapy uptake. Again, swings and roundabouts-improved penetration in larger lesions comes at the cost of lower total absorbed dose in the smaller ones.

The major outcome of this study was the finding that, rather than adopting a preloading strategy, the administration of $\alpha$-radioimmunotherapy at high specific activity, followed several hours later by a subsequent, much larger, administration of cold antibody (posttreatment dose), results in curative absorbed doses to all tumors up to $300 \mu \mathrm{m}$ in radius. The model predicts that, in the larger tumors, administration of this posttreatment dose of cold antibody saturates the easily accessible surface antigen population, improving the redistribution and penetration of the $\alpha$-radioimmunotherapy and essentially moving the binding-site barrier inward from the surface. Microtumors smaller than $50 \mu \mathrm{m}$ in radius remain susceptible to this treatment, as their readily available binding sites are saturated by the $\alpha$-radioimmunotherapy before the postloading, and the time between $\alpha$-radioimmunotherapy and posttreatment administration allows for sufficient accumulation of sterilizing $\alpha$-decays. The posttreatment cold antibody modification therefore potentially increases the overall antitumor efficacy of a given dose of $\alpha$-radioimmunotherapy, specifically targeting a disease conformation (50-300 $\mu \mathrm{m}$ in radius) previously resistant to both $\alpha$ - and $\beta$-radioimmunotherapy without additional toxicity to bone marrow.

This beneficial effect exploits the similarity between the half-life of the isotope $\left({ }^{211} \mathrm{At}, 7.2 \mathrm{~h}\right)$ and the temporal dynamics of antigen saturation, governed by the binding affinity rate constants (the on-rate and the off-rate). In the models used, antibody saturation of peripheral binding sites was maximal between 2.5 and $7.5 \mathrm{~h}$ after administration of $\alpha$-radioimmunotherapy. Given these parameters, this postloading strategy is therefore unlikely to have a major effect on the efficacy of $\alpha$-radioimmunotherapy using isotopes with longer half-lives $\left({ }^{225} \mathrm{Ac}, 10 \mathrm{~d}\right)$, and the applicability of this strategy to the $\alpha$-isotopes with very short half-lives (e.g., ${ }^{213} \mathrm{Bi}, 45 \mathrm{~min}$ ) remains to be fully examined. It is also important to keep in mind that the kinetic parameters relating to antibody concentration and initial antigen saturation rates used are likely to be specific to an intraperitoneal administration and do not necessarily apply directly to the systemic administration of antibody.

There is an ongoing debate surrounding the optimal use of radioimmunotherapy as part of a multimodal treatment strategy. Although the concept of radioisotope cocktails was proposed over 2 decades ago (2), and more recently was investigated in preclinical and limited clinical settings $(18,19)$, there are still many conceptual and regulatory roadblocks to the implementation of such a strategy. Novel targeted radiotherapies are held to a much higher standard of individual-organ dose quantification than are equivalent targeted chemotherapies, for which establishment of an empirically derived maximum tolerated dose is generally sufficient. Accurate $\alpha$-particle dosimetry is inherently complex, and it is not clear that the currently accepted organ dose tolerances, originally laid down by Emami et al. in 1991 (20), have any direct relevance to tissue response after $\alpha$ - (as opposed to $\beta$ - or photon) irradiation.

As a result of these potential hurdles, whether actual or anticipated, the theory that some combination of $\alpha$ - and $\beta$-radioimmunotherapy would produce an optimal response in patients with a spectrum of tumor sizes remains largely untested and certainty warrants a more detailed investigation as the availability of novel $\alpha$-radioimmunotherapies increases over the coming years. The findings in the study by Palm et al. (5) raise the intriguing possibility that the sequence of $\alpha$-radioimmunotherapy followed by cold antibody and subsequent $\beta$-radioimmunotherapy may provide a route to incorporating all these elements to their maximal efficacy. Apropos to the swings and roundabouts, it would appear that the maximum recipient benefit and provider profit might be obtained by optimally combining the use of both inventions.

In conclusion, whereas the use of short-half-life radioisotopes directly conjugated to intact antibodies with long circulation times has previously been considered incongruent, the study by Palm et al. (5) suggests that a timed manipulation of the $\alpha$-radioimmunotherapy-specific activity in vivo may present a way to maximize the effectiveness of this approach in treatment-resistant microtumors with minimal additional toxicity. Despite this advance, $\alpha$-radioimmunotherapy remains unlikely to prove an effective monotherapy for large solid tumors, and establishing the precise role of $\alpha$-radioimmunotherapy in a multimodal treatment plan needs to be prioritized. Accurate measures of absorbed dose in tumor and normal tissue, and the specific biologic consequences of this dose in each, are needed to better define this role. As is aptly demonstrated in this study, the use of increasingly sophisticated mathematic modeling can provide one means to design proof-ofconcept studies and derive the preclinical data required to move the field forward.

\section{DISCLOSURE}

Sean Carlin is currently supported by a generous grant from the Mesothelioma Applied Research Foundation. No other potential conflict of interest relevant to this article was reported. 


\section{REFERENCES}

1. Cobb L, Humm J. Radioimmunotherapy of malignancy using antibody targeted radionuclides. Br J Cancer. 1986;54:863-870.

2. O'Donoghue JA, Bardies M, Wheldon T. Relationships between tumor size and curability for uniformly targeted therapy with beta-emitting radionuclides. $\mathrm{J}$ Nucl Med. 1995;36:1902-1909.

3. Cheal SM, Fung EK, Patel M, et al. Curative multicycle radioimmunotherapy monitored by quantitative SPECT/CT-based theranostics, using bispecific antibody pretargeting strategy in colorectal cancer. J Nucl Med. 2017;58:1735-1742.

4. Houghton JL, Membreno R, Abdel-Atti D, et al. Establishment of the in vivo efficacy of pretargeted radioimmunotherapy utilizing inverse electron demand Diels-Alder click chemistry. Mol Cancer Ther. 2017;16:124-133.

5. Palm S, Bäck TA, Lindegren S, Hultborn R, Jacobsson L, Albertsson P. Model of intraperitoneal targeted $\alpha$-particle therapy shows posttherapy cold-antibody boost enhances microtumor radiation dose and treatable tumor sizes. $\mathrm{J} \mathrm{Nucl}$ Med. 2018;59:646-651.

6. Parker C, Nilsson S, Heinrich D, et al. Alpha emitter radium-223 and survival in metastatic prostate cancer. N Engl J Med. 2013;369:213-223.

7. Silberstein EB. Teletherapy and radiopharmaceutical therapy of painful bone metastases. Semin Nucl Med. 2005;35:152-158.

8. Sathekge M, Knoesen O, Meckel M, Modiselle M, Vorster M, Marx S. ${ }^{213} \mathrm{Bi}$ PSMA-617 targeted alpha-radionuclide therapy in metastatic castration-resistant prostate cancer. Eur J Nucl Med Mol Imaging. 2017;44:1099-1100.

9. Kratochwil C, Bruchertseifer F, Giesel FL, et al. ${ }^{225}$ Ac-PSMA-617 for PSMAtargeted $\alpha$-radiation therapy of metastatic castration-resistant prostate cancer. J Nucl Med. 2016;57:1941-1944.

10. Sutherland R, Buchegger F, Schreyer M, Vacca A, Mach J. Penetration and binding of radiolabeled anti-carcinoembryonic antigen monoclonal antibodies and their antigen binding fragments in human colon multicellular tumor spheroids. Cancer Res. 1987;47:1627-1633.
11. Fujimori K, Covell DG, Fletcher JE, Weinstein JN. A modeling analysis of monoclonal antibody percolation through tumors: a binding-site barrier. $\mathrm{J} \mathrm{Nucl}$ Med. 1990;31:1191-1198.

12. O'Donoghue JA. Implications of nonuniform tumor doses for radioimmunotherapy. J Nucl Med. 1999;40:1337-1341.

13. Cederkrantz E, Andersson H, Bernhardt P, et al. Absorbed doses and risk estimates of ${ }^{211}$ At-MX35 $\mathrm{F}\left(\mathrm{ab}^{\prime}\right) 2$ in intraperitoneal therapy of ovarian cancer patients. Int J Radiat Oncol Biol Phys. 2015;93:569-576.

14. Andersson H, Cederkrantz E, Back T, et al. Intraperitoneal alpha-particle radioimmunotherapy of ovarian cancer patients: pharmacokinetics and dosimetry of ${ }^{211}$ At-MX35 F(ab')2—a phase I study. J Nucl Med. 2009;50:1153-1160.

15. Garkavij M, Tennvall J, Strand SE, et al. Enhanced radioimmunotargeting of ${ }^{125}$ I-labeled L6-biotin monoclonal antibody (MAb) by combining preload of cold L6 MAb and subsequent immunoadsorption in rats. Cancer Res. 1995;55:5874s5880s.

16. Buchsbaum DJ, Wahl RL, Glenn SD, Normolle DP, Kaminski MS. Improved delivery of radiolabeled anti-B1 monoclonal antibody to Raji lymphoma xenografts by predosing with unlabeled anti-B1 monoclonal antibody. Cancer Res. 1992;52:637-642.

17. Witzig TE, Fishkin P, Gordon LI, et al. Treatment recommendations for radioimmunotherapy in follicular lymphoma: a consensus conference report. Leuk Lymphoma. 2011;52:1188-1199.

18. de Jong M, Breeman WA, Valkema R, Bernard BF, Krenning EP. Combination radionuclide therapy using ${ }^{177} \mathrm{Lu}$ - and ${ }^{90} \mathrm{Y}$-labeled somatostatin analogs. $J$ Nucl Med. 2005;46(suppl 1):13S-17S.

19. Dumont RA, Seiler D, Marincek N, et al. Survival after somatostatin based radiopeptide therapy with ${ }^{90}$ Y-DOTATOC vs. ${ }^{90}$ Y-DOTATOC plus ${ }^{177} \mathrm{Lu}-$ DOTATOC in metastasized gastrinoma. Am J Nucl Med Mol Imaging. 2014;5: 46-55.

20. Emami B, Lyman J, Brown A, et al. Tolerance of normal tissue to therapeutic irradiation. Int J Radiat Oncol Biol Phys. 1991;21:109-122. 